\title{
Malignancy transformation of chronic osteomyelitis: description of 6 cases of Marjolin's ulcers
}

\author{
Julia Steinrücken • Maria-Chiara Osterheld • \\ Andrej Trampuz $\cdot$ Olivier Borens
}

Received: 16 September 2010 / Accepted: 22 March 2011 / Published online: 12 April 2011

(C) Springer-Verlag 2011

\begin{abstract}
Background Marjolin's ulcer describes any malignant transformation of a chronic inflammatory lesion. In the majority of cases, a squamous cell carcinoma is diagnosed. Malignant transformation occurs usually after a long period of latency of chronic infection; it takes approximately 35 years on average. There are no typical clinical presentations, but several indirect signs may suggest the malignant transformation, such as increased or changed discharge, pathologic fracture, a slow-growing exophytic mass, or other suggestive signs of malignant transformation, which should prompt to biopsy for histological exam. The diagnosis of chronic osteomyelitis should not prevent to search for carcinoma.

Case description We present six patients with chronic osteomyelitis that developed well-differentiated squamous cell carcinoma. All patients were older than 50 years (mean 60 years, range 52-77 years). Five Marjolin's ulcers were located on the lower limb and one on the arm. The average time of the chronic discharging osteomyelitis before diagnosis
\end{abstract}

J. Steinrücken · A. Trampuz

Infectious Diseases Service, University Hospital,

Lausanne, Switzerland

M.-C. Osterheld

Institute of Pathology, University Hospital, Lausanne, Switzerland

O. Borens $(\bowtie)$

Departement of Orthopaedics and Trauma Surgery,

Septic Unit, University and University Hospital of Lausanne

(CHUV), Rue du Bugnon 46, 1011 Lausanne, Switzerland

e-mail: olivier.borens@chuv.ch of carcinoma ranged between 12 and 40 years. All patients were treated by amputation of the affected limb. None had metastasis, and one patient developed local recurrence and received palliative treatment.

Clinical relevance Our study emphasizes that Marjolin's ulcer should be considered as a rare but significant longterm complication of chronic osteomyelitis. The finding of microorganisms should not prevent from further diagnostic procedures by histopathological examination so that the correct surgical treatment can be performed.

Keywords Chronic osteomyelitis - Squamous cell carcinoma $\cdot$ Microbiology $\cdot$ Pathology $\cdot$ Orthopedics

\section{Introduction}

Malignant changes associated with burns, scars, chronic ulcers and draining osteomyelitis are well known since over a century. The French surgeon Jean-Nicolas Marjolin first reported in 1928 a case of cellular changes of ulcerated lesions of vascular origin, i.e., Marjolin's ulcer. Ten years later, Hawkins described a squamous cell carcinoma, which developed in long-standing chronic osteomyelitis patient $[1,2]$. In 1963, after the publication of Sedlin and Fleming's [3] series, the name Marjolin's ulcer was also applied to neoplastic transformations with onset on sites of chronic bone infection.

Today the term Marjolin's ulcer designates any malignant transformation of a chronic inflammatory skin lesion, whatever the origin of the lesion and whatever type of cancer develops in the lesion. In the majority of the cases, this is a squamous cell carcinoma, and other tumors that may be found are for example myeloma, sarcoma, and lymphoma [3-7]. The incidence of malignant transformation in 
chronic osteomyelitis is reported between 0.2 and $1.7 \%$ of cases of chronic osteomyelitis [8-13].

Malignant transformation occurs usually after a long period of latency of chronic infection; it takes approximately 35 years on average [3]. Squamous cell carcinoma is also in patients with chronic osteomyelitis the most common cancer. Usually the squamous cell carcinoma occurs in areas exposed to the sun. The risk of metastasis and recurrence rate is low, except cancers of the lip and ears [11]. Although the malignant alteration of a chronic wound is well known to dermatologists, Marjolin's ulcer associated with chronic osteomyelitis is often diagnosed with delay. For final diagnosis, a deep biopsy at the site of inflammation in the ulcer ground or fistula is needed. A superficial biopsy might be misinterpreted as a chronic inflammation with actinic keratosis. Invasive squamous cell carcinoma refers to cancer cells that have grown into the deeper layers of the skin, the dermis, or bone. They can generally be treated by local excision or amputation. Occasionally, nonsurgical treatment options are topical chemotherapy, topical immune response modifiers, photodynamic therapy, radiotherapy, and systemic chemotherapy $[8,11]$.

Few cases of well-differentiated squamous cell occurring in chronic osteomyelitis patients have been reported [3, 1417]. We report six additional cases of Marjolin's ulcers associated with chronic cutaneous lesions related to underlying osteomyelitis that were observed between 1985 and 2009 in our orthopaedic department. The importance of correct diagnosis is emphasized since a misdiagnosis for a chronic infection may delay patient management and worsen outcome.

\section{Case presentations}

Case 1

A 62-year-old Turkish woman presented with chronic osteomyelitis of the right proximal humerus persisting for 40 years. The patient presents with recent development of three discharging sinuses and ulcerations of the proximal third of the arm. Staphylococcus aureus and Escherichia coli were found in wound cultures. A biopsy specimen was taken from the right proximal humerus and revealed a highly-differentiated squamous cell carcinoma invading bone. Proximal amputation of the arm was performed with a macroscopically complete resection. Unfortunately, microscopic examination of the surgical specimen showed that the proximal margin was positive for tumor. The patient received no additional treatment. Three years later, the tumor recurred close at the site of previous surgery with infiltration of scapula and thoracic soft tissues. A palliative surgical excision of the tumor was performed, and the patient was discharged. She returned to her home country and was lost to follow-up.
Fig. 1 Patient $\mathrm{N}^{\circ} 5$ a Ulcerated lesion of the distal left leg showing irregular contours and slightly elevated edges. b Longitudinal section showing a whitish «cheesy» necrotic tumor infiltrating deeply inside soft tissues and tibial bone
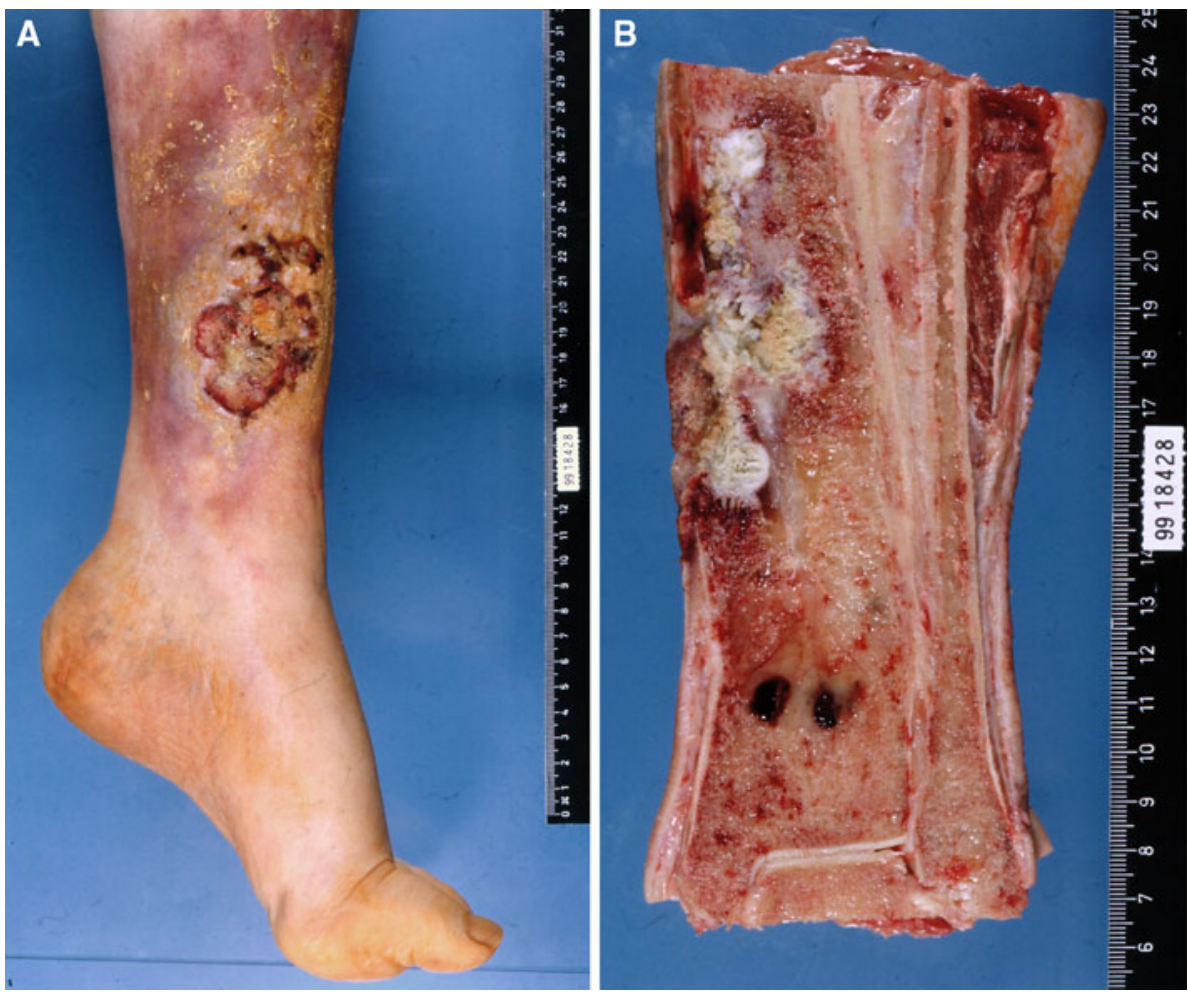


\section{Case 2}

A 54-year-old woman was operated for a bimalleolar fracture of the left ankle in 1976. Since operation, she complained about pain in the left foot and was able to walk only on her heel. First cutaneous ulceration with discharge occurred 9 years later and progressed in size. Because of development of a chronic ulceration and a sinus tract, she was regularly followed up over the years, but histology showed no signs of malignancy. In May 1987, the ulceration was surgically excised. One year later, in 1988, osteomyelitis caused by Proteus mirabilis and Klebsiella spp. was diagnosed with concomitant significant bone destruction. An amputation of the left foot was performed. On histological examination, in addition to chronic osteomyelitis, a well-differentiated squamous cell carcinoma of the sinus tract was diagnosed, invading the adjacent soft tissues and the underlying bone. The postoperative course was uneventful. The patient was referred to her physician and showed no recurrence of infection or tumor at follow-up after 1 year.

\section{Case 3}

A 59-year-old man was admitted in 1975 after a road accident with fractures of left femur, left tibia, and ribs. At the tibia, a preexisting chronic osteomyelitis was known since the age of 4 years with persisting ulceration, for which he underwent 15 surgeries without cure. One year after the accident, a chronic osteomyelitis caused by $S$. aureus was diagnosed, and a sequestrectomy and skin grafting with antibiotic therapy was performed. A chronic ulceration persisted the following 12 years. In 1988, he was admitted because of complete obstruction of the femoral artery, and a thromboendarterectomy was carried out with debridement and additional bone and skin grafts. After this surgery, a discharging sinus occurred and persisted for 8 years; when in 1996, he presented with a painful swelling of the tibial crest above the discharging sinus. Deep biopsies from the edges of the sinus tract revealed a well-differentiated squamous cell carcinoma infiltrating the underlying soft tissues and bone. Bone cultures grew E. coli. A lower limb amputation was performed. Postoperative outcome was uneventful after 6 months.

\section{Case 4}

A 52-year-old patient was admitted in 1998 for acute pain in the area of a nonhealing ulcer of the right lower limb. The ulcer persisted since the age of 19 years, after a fracture of the tibia. Eleven years before admission, he developed a cutaneous sinus overlying an osteomyelitis that was treated with a sequestrectomy, which was uneventful for 9 years, when a sinus reappeared in 1996 with development of an

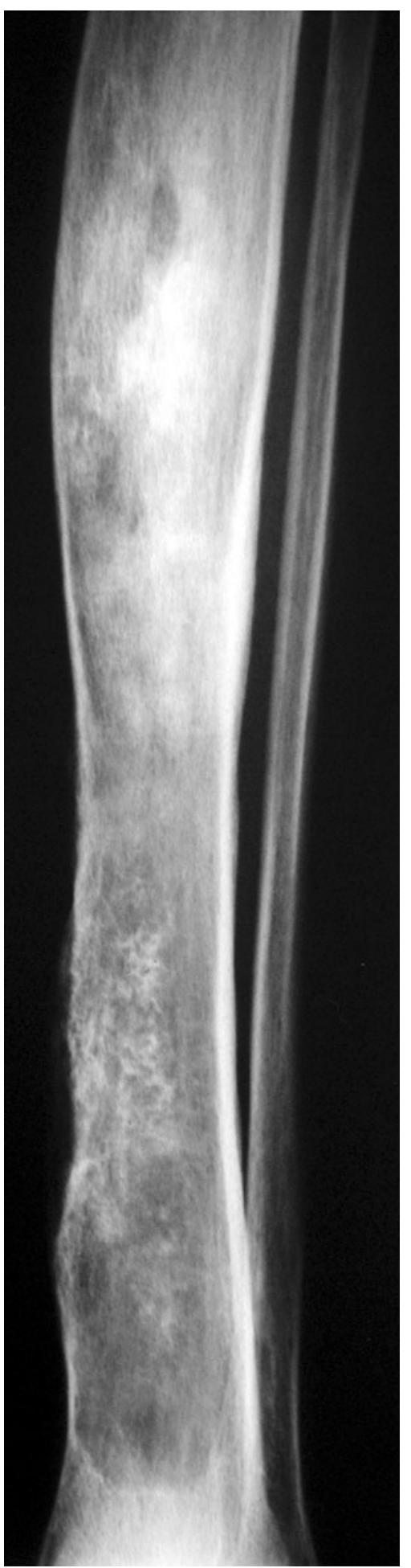

Fig. 2 Patient $\mathrm{N}^{\circ} 5$ in the radiograph the lesions present as gray, whitish, exophytic, partially hemorrhagic, or necrotic tumors

abscess 1 year later. A sequestrectomy was performed. At current hospitalization, a pathological fracture of the fibula was treated by internal fixation. Intraoperative biopsies grew S. epidermidis and Peptostreptococcus spp., which were 
treated with antibiotics. Because of a persisting discharging sinus, another sequestrectomy was performed. Histopathology showed a well-differentiated squamous cell carcinoma and chronic osteomyelitis. Amputation of the right leg was performed. At last follow-up examination, 3 years later, there was neither local recurrence nor distant metastasis.

\section{Case 5}

A 56-year-old Italian man was admitted in 2001 because of a discharging sinus of the left leg that appeared 3 months before and did not respond to antibiotic therapy. Figure 1 shows the macroscopic picture of the exophytic tumor with a discharging sinus (Fig. 1a and b). The radiograph showed a gray, partially hemorrhagic or necrotic tumors (Fig. 2). Past medical history revealed surgeries in 1972 and 1976 for a fracture of the left leg because of persisting chronic osteomyelitis for 30 years. A bone biopsy was taken from the ulcer ground showed acute and chronic osteomyelitis and differentiated squamous cell carcinoma, which infiltrated the underlying bone. Microbiology showed mixed skin flora. Aware of his diagnosis and prognosis, the patient decided to return to his maternal country and was lost to follow-up.

\section{Case 6}

A 77-year-old man was referred to our institution in 2009 for a surgical treatment of a nonhealing chronic discharging fistula of the proximal right tibia occurring after a sport accident 39 years ago. He underwent a surgical debridement with removal of the fistula and sequestrectomy and placement of a vancomycin-impregnated spacer, cutaneous closure of the wound. Tissue cultures grew S. aureus and Streptococcus agalacticae treated with intravenous antibiotics. Histological examinations revealed a well-differentiated squamous cell carcinoma invading the bone. Distal amputation with macro- and microscopically complete tumor resection of the upper leg was performed 1 month after diagnosis. The postoperative course was uncomplicated with uneventful wound healing and follow-up.

\section{Discussion}

Although considered as a rare complication of chronic osteomyelitis, carcinomas and sarcomas may occur in chronic draining sinuses of osteomyelitis [4-7, 12], a well-known phenomena since over a century. In the current study, we describe 6 cases of well-differentiated squamous cell carcinoma, which developed in long-standing chronic osteomyelitis. In most cases, these types of malignancy occur in men during the fifth and sixth decades of life, usually after a long history (20-30 years) of chronic osteomyelitis with intermittently discharging sinuses [14, 17]. All 6 patients in this case series had a long history of relapsing bone infection ranging from 12 to 40 years, and five of them have been operated several times. Congruent to the literature [14, 17], in five of six patients, the chronic osteomyelitis was localized in the lower limb.

Table 1 Characteristics of six patients with well-differentiated squamous cell carcinoma developed in chronic osteomyelitis

\begin{tabular}{|c|c|c|c|c|c|c|}
\hline Patient & 1 & 2 & 3 & 4 & 5 & 6 \\
\hline Sex & Male & Female & Female & Male & Male & Male \\
\hline Age (years) & 62 & 54 & 59 & 52 & 56 & 77 \\
\hline Anatomic site & Humerus & Tibia + Fibula & Tibia & Tibia + Fibula & Tibia & Tibia \\
\hline Duration (years) & 40 & 12 & 21 & 19 & 30 & 39 \\
\hline $\begin{array}{l}\text { Number } \\
\text { of surgeries }\end{array}$ & 0 & 4 & 19 & 5 & 6 & 4 \\
\hline Clinical symptoms & Sinus & Sinus & Sinus & Sinus & Sinus & Sinus \\
\hline Microbiology & $\begin{array}{l}\text { S.aureus } \\
\text { E.coli }\end{array}$ & $\begin{array}{l}\text { P.mirabilis } \\
\text { Klebsiella spp. }\end{array}$ & E.coli & $\begin{array}{l}\text { S.epidermidis } \\
\text { Peptostrepto coccus }\end{array}$ & Mixed skin flora & $\begin{array}{l}\text { S.aureus } \\
\text { S.agalacticae }\end{array}$ \\
\hline Histopathology & $\begin{array}{l}\text { Well-differentiated } \\
\text { SCC* }\end{array}$ & $\begin{array}{l}\text { Well-differentiated } \\
\text { SCC* }\end{array}$ & $\begin{array}{l}\text { Well-differentiated } \\
\text { SCC* }\end{array}$ & $\begin{array}{l}\text { Well-differentiated } \\
\text { SCC* }\end{array}$ & $\begin{array}{l}\text { Well-differentiated } \\
\text { SCC* }\end{array}$ & $\begin{array}{l}\text { Well-differentiated } \\
\text { SCC* }\end{array}$ \\
\hline Bone invasion & Yes & Yes & Yes & Yes & Yes & Yes \\
\hline Metastases & No & No & No & No & No & No \\
\hline $\begin{array}{l}\text { Local } \\
\quad \text { recurdescence }\end{array}$ & Yes & No & No & No & No & No \\
\hline Treatment & Amputation & Amputation & Amputation & Amputation & Unknown & Amputation \\
\hline
\end{tabular}

* SCC squamous cell carcinoma 
Table 1 summarizes the characteristics of our patients. All our patients had a draining sinus, indicating a chronic inflammation. Diagnosis of chronic osteomyelitis is often based on radiologic results and microbiology [11]. All our patients with squamous cell carcinoma had a concomitant chronic osteomyelitis, with similar pathogens isolated in culture biopsy from the bone as reported in the literature [18]. Most common organisms are S. aureus and streptococci, enterococci, Gram-negative rods, and anaerobes.

Well-differentiated squamous cell carcinoma was diagnosed in histopathology in all cases with infiltration of the skin and the bone, with a concomitant acute on chronic inflammation. Squamous cell carcinoma is thought to arise from the epidermal opening line of the fistula tract, and it would extend secondarily in the deep soft tissues of the limb and the underlying infected bone. Pathological finding as the frequent presence of a marked epidermal hyperplasia around the fistula opening supports this hypothesis. Definite carcinogenic factors in chronic osteomyelitis have not yet been identified, but long-standing and persistent action of various cytokines related to the inflammatory context on basal epidermal cells is likely responsible for the malignant transformation. Therefore, usually long duration of inflammation precedes the development of the carcinoma. Differentiating a pseudoepitheliomatous hyperplasia from a highly differentiated epidermoid carcinoma is difficult for the pathologist, especially if small or superficial biopsies were collected. It is important to take multiple deep tissue fragments from sinus tract edges [15].

In this report, we presented six cases of well-differentiated squamous cell carcinoma, which occurred in the setting of chronic osteomyelitis. Malignant transformation of epithelial cells in chronic osteomyelitis with discharging sinus is a rare but serious event that may appear at any time in the course of the disease but most often after 10 or more years. It should be suspected in case of increased or changed discharge, pathologic fracture, a slow-growing exophytic mass or other suggestive signs of malignant transformation, which should prompt to biopsy for histological exam. Surgical resection (most often amputation) is the treatment of choice.

Conflict of interest The authors declare that they have no financial relation with regard to this article. We also state that we had full control of all primary data, and we agree to allow the journal to review our data if requested.

\section{References}

1. Hawkins C (1835) Cases of warty tumors in cicatrises. Medical chirurgie transactions 19:19

2. Marjolin JD (1981) Development of carcinomatous ulcers in scars. Foot note. Bailey and Love's Short Practice of Surgery, 24th edn. H. K. Lewis Co., Ltd, London, p 120

3. Rieger UM, Kalbermatten DF, Wettstein R, Heider I, Haug M, Pierer G (2008) Marjolin's ulcer revisited-basal cell carcinoma arising from grenade fragments? Case report and review of the literature. J Plastic Reconstruct Aesthet Surg 61:65-70

4. Johnston RM, Miles JS (1973) Sarcomas arising from chronic osteomyelitic sinuses. J Bone Joint Surg 55:162-168

5. Kennedy C, Stoker DJ (1990) Case report: malignant fibrous histiocytoma complicating chronic osteomyelitis. Clin Radiol 41:435-436

6. Kirshbaum JD (1949) Fibrosarcoma of the tibia following chronic osteomyelitis: report of a case. J Bone Joint Surg 31:413-416

7. Lidgren L (1973) Neoplasia in chronic fistulating osteitis. Acta Orthop Scand 44:152-156

8. Bauer T, David T, Rimareix F, Lortat-Jacob A (2007) Marjolin's ulcer in chronic osteomyelitis: seven cases and a review of the literature. Revue chirurgie Orthopédique 93:63-71

9. Blidi M, Gatefosse M, Barjonnet G, Bedoucha JS, Wajcner G (1996) Carcinome épidermoïde compliquant une ostéomyélite chronique du fémur. Revue du Rhumatisme 63:67-69

10. Bolsen B (1981) Soft tissue sarcoma of limbs: better out-look. JAMA 246:2007-2008

11. Kumar V, Abbas AK, Fausto N, Mitchell R (2007) Robbins Basic Pathology, 8th edn. Saunders Elsevier, Philadelphia, pp 810-811

12. Sadat-Ali M, Geeranavar SS (1996) Malignancy in chronic osteomyelitis sinus. Ind J Cancer 33:139-144

13. Sankaran-Kutty MS, Corea JR, Ali MS, Kutty MK (1985) Squamous cell carcinoma in chronic osteomyelitis: report of a case and review of the literature. Clin Orthop 198:264-267

14. Lifeso RM, Bull CA (1985) Squamous cell carcinoma of the extremities. Cancer 55:2862-2867

15. Noonan KJ, Goetz DD, Marsh JL, Peterson KK (1993) Rapidly destructive squamous cell carcinoma as a complication of chronic osteomyelitis. Orthopedics 16:1140-1144

16. Wagner RF, Grande DJ (1986) Pseudoepitheliomatous hyperplasia vs. squamous cell carcinoma arising from chronic osteomyelitis of the humerus. J Dermat Surg Oncol 12:632-635

17. Weng-Neng U, Fu-Chan W, Swei H, Chun-Hsiung S (1993) Squamous cell carcinoma complicating tibial osteomyelitis treated with local wide excision and staged microvascular reconstruction. Clin Orthop Relat Res 293:274-279

18. Senneville E, Morant H, Descamps D et al (2009) Needle puncture and transcutaneous bone biopsy cultures are inconsistent in patients with diabetes and suspected osteomyelitis of the foot. Clin Infect Dis 48:888-893 